\title{
NONPARAMETRIC ESTIMATION FOR A COMPOUND POISSON PROCESS GOVERNED BY A MARKOV CHAIN
}

UDC 519.21

\author{
O. A. VOǏNA
}

\begin{abstract}
The problem of nonparametric estimation for a Poisson process governed by a Markov chain with continuous time is considered in the case of incomplete observations. A method of the nonparametric estimation is proposed. The method is based on a representation of such a process in the form of a hidden Markov model. An example for the estimation of unknown distribution functions is considered in the case of incomplete observations after a compound Poisson process governed by a simple Markov regenerative process.
\end{abstract}

\section{INTRODUCTION}

Consider an example of a multidimensional stochastic process with a discrete interference of chance. The model considered in the paper can be described by the words "compound Poisson process governed by a Markov chain with continuous time". Processes of this type provoke a permanent interest, since they have a pronounced applied character. Specific interpretations of their structural elements create wide class of applications for studies of various natural phenomena. For example, the processes studied in 2 can effectively be applied for optimal risk control in models of logistics management as well as in those of insurance, etc. The number of publications devoted to various classes of processes with a discrete interference of chance is practically limitless.

The models considered in the current paper are also studied in [1, 3, 4, 6, 7]. The main aim of [1, 3, 4, 6, 7] is to give rigorous definitions of stochastic processes with a discrete interference of chance, to investigate the distributions of some functionals of such processes and their limit behavior, and to approximate the processes by simpler models under the condition that a structure of the process is well defined.

In a certain sense, the current paper solves an inverse problem, since we recover the elements of the "stochastic structure" of a process with discrete interference of chance from observations after functionals of its trajectories. We assume that the values of the processes are unknown and are not available for observations. The corresponding stochastic data and related methods are known under the name of "hidden Markov models" (see [8, 9, 10]).

\section{Mainstream}

Assume that the following three random objects are defined in the same probability space $(\Omega, \Im, P)$ :

2010 Mathematics Subject Classification. Primary 62M05; Secondary 60J99, 93E11.

Key words and phrases. Compound Poisson process, incomplete observations, nonparametric estimation, hidden Markov model, Cox model. 
(1) A Markov chain $x(t), t \geq 0$, with continuous time, with the set of states

$$
E=\{1,2, \ldots, m\},
$$

and with intensities of transition defined by the following matrix

$$
A=\left\|a_{i j}\right\|, \quad i, j=1,2, \ldots, m .
$$

(2) For every state $i \in E$, a random variable $\zeta_{i}$ with the distribution function

$$
U_{i}(x)=\mathrm{P}\left\{\zeta_{i}<x\right\}, \quad i \in E=\{1,2, \ldots, m\},
$$

is defined.

(3) A Poisson process $\Pi(t), t \geq 0$, with parameter $\lambda$ that does not depend on the chain $x(t)$ and on the family of random variables $\left\{\zeta_{i}, i \in E\right\}$.

Assume that a point stochastic process $S(n) \in[0,+\infty)$ with discrete time $n=$ $0,1,2, \ldots$ describes the sequential jumps of the Poisson process $\Pi(t)$, that is

$$
S(0)=0, \quad \Pi(t)=\max \{k: S(k) \leq t\} .
$$

Consider a family of random variables $\left[\left\{\zeta_{i}^{(k)}, k=0,1,2, \ldots\right\}, i \in E\right]$ being independent of stochastic processes $x(t)$ and $\Pi(t)$ and such that, for different values of the index $k$, the random variables $\zeta_{i}^{(k)}$ are independent, their distribution does not depend on the index $k$, and is determined by the distribution functions $U_{i}(x), i=1,2, \ldots, m$.

Definition 2.1. A stochastic vector process

$$
\xi(t)=\left\{\xi_{1}(t), \xi_{2}(t), \ldots, \xi_{m}(t)\right\}, \quad t \geq 0,
$$

is called a multidimensional compound Poisson process governed by a Markov chain with continuous time if

$$
\begin{gathered}
\xi_{x(S(k))}(t)=\xi_{x(S(k))}(S(k))+\zeta_{x(S(k))}^{(k)}, \quad S(k)<t \leq S(k+1) ; \\
\xi_{j}(t)=\xi_{j}(S(k)), \quad j \neq x(S(k)), \quad S(k)<t \leq S(k+1) .
\end{gathered}
$$

Consider the following stochastic process

$$
\zeta(k)=\zeta_{x(S(k))}^{(k)}, \quad k=1,2,3, \ldots
$$

According to [8, 9, the process $\zeta(k), k=1,2,3, \ldots$, can be viewed as a special representative of a "hidden Markov model". It is shown in 2 that this stochastic process is one of the coordinates of a "hidden Markov model" $(\zeta(k), \eta(k)), k=0,1,2, \ldots$, where $\eta(k)$, $k=0,1,2, \ldots$, is a certain Markov chain. The transition probabilities of the "hidden" chain are also found in 2 .

Consider the problem of nonparametric estimation for the model described above in the case of incomplete statistical information. Namely we assume that the stochastic process $\zeta(k), k=1,2,3, \ldots$, is observable and let

$$
\{\zeta(1), \zeta(2), \ldots, \zeta(N)\}
$$

be the vector of its $N$ sequential values. The values of the Markov chain $x(t), t \geq 0$, and those of the Poisson process $\Pi(t), t \geq 0$, are unknown in the intervals of observations. However we know their stochastic structure, that is we assume that the matrix $A=\left\|a_{i j}\right\|$, $i, j=1,2, \ldots, m$, vector $\pi=\left\{\pi_{1}, \pi_{2}, \ldots, \pi_{m}\right\}$ of the initial distribution of the Markov chain $x(t), t \geq 0$, and parameter $\lambda$ of the Poisson process $\Pi(t), t \geq 0$, are known.

Let $a$ be some fixed real number. Consider the vector

$$
u^{(a)}=\left\{u_{1}^{(a)}, u_{2}^{(a)}, \ldots, u_{m}^{(a)}\right\},
$$

where

$$
u_{i}^{(a)}=U_{i}(a)=\mathrm{P}\left\{\zeta_{i}<a\right\}, \quad i \in E=1,2, \ldots, m,-\infty<a<+\infty .
$$


Our main aim is to construct consistent and asymptotically normal estimators $\hat{u}_{n}^{(a)}$ for the vector $u(a)$ from the vector of observations

$$
\{\zeta(1), \zeta(2), \ldots, \zeta(n), \zeta(n+1), \ldots, \zeta(n+m)\} .
$$

A method of statistical estimation of parameters of hidden Markov models is proposed in [10]. An example of an application of this method is given in 11. We will use results of the paper [10] and representation of the process $\zeta(k), k=1,2,3, \ldots$, in terms of a "hidden Markov model" (see [2]) to solve the problem of the nonparametric estimation described above.

Let $A$ be a random event. The symbol $\chi[A]$ stands for the indicator of an event $A$. Consider the family of statistics

$$
\left\{Q_{s n}(y, z), s=1,2, \ldots, m\right\}, \quad n=1,2, \ldots,
$$

defined by

$$
Q_{s n}(y, z)=\frac{1}{n} \sum_{t=1}^{n} \chi[\zeta(t)<y, \zeta(t+s)<z], \quad s=1,2, \ldots, m,
$$

where $y$ and $z$ are arbitrary fixed real numbers. Further let $\left\{q_{s n}^{(a)}, s=1,2, \ldots, m\right\}$, $n=1,2, \ldots$, be a special case of a family of statistics $\left\{Q_{s n}(y, z)\right\}$ corresponding to $y=z=a$, namely

$$
q_{s n}^{(a)}=Q_{s n}(a, a), \quad s=1,2, \ldots, m .
$$

On the main probability space $(\Omega, \Im, \mathrm{P})$, consider a discrete homogeneous Markov chain $\eta(k), k=0,1,2, \ldots$, with the set of states $E=\{1,2, \ldots, m\}$ that does not depend on the chain $x(t)$, on the Poisson process $\Pi(t)$, and on the family of random variables $\left\{\zeta_{i}, i \in E\right\}$. The one step transition probabilities

$$
P=\left\|p_{i j}\right\|, \quad i, j=1,2, \ldots, m,
$$

of the chain $\eta(k)$ can be evaluated from the system of linear equations

$$
\begin{gathered}
p_{i j}-\delta_{i j}=\sum_{k=1}^{m} p_{i k} \cdot \frac{a_{k j}}{\lambda}, \\
i=1,2, \ldots, m, \quad j=1,2, \ldots, m,
\end{gathered}
$$

where

$$
\delta_{i j}=1, i=j, \quad \delta_{i j}=0, i \neq j
$$

Let

$$
P(s)=\left\|p_{i j}^{(s)}\right\|=P^{s}
$$

denote the matrix of transition probabilities of the Markov chain $\eta(k)$ over $s$ steps.

Theorem 2.1. Assume that

1) the Markov chain $\eta(k), k=0,1,2, \ldots$ is irreducible and aperiodic;

2) the eigenvalues $\left\{b_{1}, b_{2}, \ldots, b_{m}\right\}$ of the matrix $P$ are all different and nonzero;

3) for all $i=1,2, \ldots, m$ and $j=1,2, \ldots, m$,

$$
\sum_{k=1}^{m} \frac{\pi_{k}}{\sqrt{\pi_{i} \cdot \pi_{j}}} \cdot p_{k i} \cdot p_{k j}=\sum_{k=1}^{m} \frac{\sqrt{\pi_{i} \cdot \pi_{j}}}{\pi_{k}} \cdot p_{i k} \cdot p_{j k},
$$

where $\pi=\left\{\pi_{1}, \pi_{2}, \ldots, \pi_{m}\right\}$ is the vector of the stationary distribution of the Markov chain $\eta(k)$. 
Then the system of equations

$$
\sum_{i=1}^{m} \sum_{j=1}^{m} \pi_{i} \cdot p_{i j}^{(s)} \cdot u_{i} \cdot u_{j}=q_{s n}^{(a)}, \quad s=1,2, \ldots, m,
$$

has real solutions with a probability converging to 1 as $n \rightarrow \infty$.

Proof. Theorem 2.1 follows from the results of the papers [10] and [2].

It is shown in the paper [10] that condition (1) for the Markov chain $\eta(k)$ in a hidden Markov model $(\zeta(k), \eta(k)), k=0,1,2, \ldots$, implies the solvability of the system of equations (2); another result of [10] is the method developed to find the solutions of system (2). According to [10, one of the solutions of system (2) is a consistent estimator of the vector

$$
u^{(a)}=\left\{u_{1}^{(a)}, u_{2}^{(a)}, \ldots, u_{m}^{(a)}\right\} .
$$

Therefore we follow the method described in [10] for solving the system of equations (2) and for searching a consistent estimator

$$
\hat{u}_{n}^{(a)}=\left\{\hat{u}_{n 1}^{(a)}, \hat{u}_{n 2}^{(a)}, \ldots, \hat{u}_{n m}^{(a)}\right\}
$$

of the vector $u^{(a)}$ in the set of solutions of system (2).

Some other properties of estimators obtained from the system of equations (2) are studied in the paper [10]. For example, according to [10], the estimator $\hat{u}_{n}^{(a)}$ defined in Theorem 2.1 is asymptotically normal.

Since the model under investigation has many parameters and since the estimation method described in Theorem 2.1 is rather complicate, a detailed analysis of the statistical procedure and studies of properties of estimators is almost impossible. Theorem 2.1 however indicates a way to solve these problems by using the results of [10]. It seems reasonable to study the estimators in a specific case where the true values of the parameters of the model are known.

The rest of the paper is devoted to an application of the method of Theorem 2.1 for the estimation of unknown distribution functions of a compound Poisson process governed by a simple Markov regenerative process if the statistical information is incomplete. In other words, we consider a particular case of the model and show how the statistical procedure works in a simplest situation where $m=2$.

First we consider the problem of statistical estimation of unknown distribution functions in a Cox model [5]. Let $(c(t), \tau(t))$ be a stochastic process with discrete time and assume that its values belong to $\{1,2\} \times(-\infty, \infty)$. We assume that $c(t) \in\{1,2\}$ is a homogeneous Markov chain with a matrix $C$ of one step transition probabilities

$$
C=\left\|c_{i j}\right\|, \quad i \in\{1,2\}, j \in\{1,2\},
$$

where

$$
\begin{aligned}
& c_{11}=1-\alpha, \quad c_{12}=\alpha, \\
& c_{21}=\beta, \quad c_{22}=1-\beta, \\
& 0<\alpha<1, \quad 0<\beta<1,
\end{aligned}
$$

and where $\tau(t)$ is a process of conditionally independent random variables defined on the Markov chain $c(t)$, that is

$$
\tau(t)=\tau_{c(t)}^{(t)}, \quad t=1,2, \ldots
$$

Here $\left\{\tau_{i}^{(t)}, i \in\{1,2\}, t=1,2, \ldots\right\}$ is a family of random variables being independent of the chain $c(t)$, being mutually independent for different $t=1,2, \ldots$, and for which the 
distribution functions

$$
T_{i}(x)=\mathrm{P}\left\{\tau_{i}^{(t)}<x\right\}, \quad t=1,2, \ldots,
$$

do not depend on the index $t=1,2, \ldots$. Let $c=\left\{c_{1}, c_{2}\right\}$ be the vector of the stationary distribution of the chain $c(t)$, that is

$$
c_{1}=\frac{\beta}{\alpha+\beta}, \quad c_{2}=\frac{\alpha}{\alpha+\beta},
$$

and let $\{1, b\}$ be the vector of eigennumbers of the matrix $C$, where

$$
b=1-\alpha-\beta \text {. }
$$

Consider the functions

$$
\begin{gathered}
T^{(0)}(x)=c_{1} \cdot T_{1}(x)+c_{2} \cdot T_{2}(x), \\
T^{(1)}(x, y)=\sum_{i=1}^{2} \sum_{j=1}^{2} c_{i} \cdot c_{i j} \cdot T_{i}(x) \cdot T_{j}(y), \\
T^{(2)}(x, y)=\sum_{i=1}^{2} \sum_{j=1}^{2} c_{i} \cdot c_{i j}^{(2)} \cdot T_{i}(x) \cdot T_{j}(y),
\end{gathered}
$$

where

$$
C^{(2)}=C^{2}=\left\|c_{i j}^{(2)}\right\|, \quad i \in\{1,2\}, j \in\{1,2\},
$$

are the two step transition probabilities of the Markov chain $c(t)$. Put

$$
\begin{aligned}
& T^{(1)}(x)=T^{(1)}(x, x), \\
& T^{(2)}(x)=T^{(2)}(x, x) .
\end{aligned}
$$

Now let

$$
\{\tau(1), \tau(2), \ldots, \tau(n), \tau(n+1), \tau(n+2)\}
$$

be the vector of observations after the process $\tau(t)$ in the interval $t \in\{1,2, \ldots, n+2\}$. Using these observations, we introduce the following statistics:

$$
\begin{gathered}
\hat{T}_{n}^{(1)}(x, y)=\frac{1}{n} \sum_{t=1}^{n} \chi[\tau(t)<x, \tau(t+1)<y], \\
\hat{T}_{n}^{(2)}(x, y)=\frac{1}{n} \sum_{t=1}^{n} \chi[\tau(t)<x, \tau(t+2)<y], \\
\hat{T}_{n}^{(0)}(x)=\frac{1}{n} \sum_{t=1}^{n} \chi[\tau(t)<x] .
\end{gathered}
$$

Let

$$
\hat{T}_{n}^{(1)}(x)=\hat{T}_{n}^{(1)}(x, x), \quad \hat{T}_{n}^{(2)}(x)=\hat{T}_{n}^{(2)}(x, x) .
$$

It is easy to check that condition (1) of Theorem 2.1 holds for the Markov chain $c(t) \in\{1,2\}$ whose set of states consists of two elements. Let $z$ denote the vector-column with the coordinates $z_{1}$ and $z_{2}$, and let the symbol $\Delta$ stand for the diagonal matrix formed by numbers $c_{1}$ and $c_{2}$ at its diagonal, that is

$$
\Delta=\operatorname{diag}\left\{c_{1}, c_{2}\right\} \text {. }
$$

Then the system of equations (2) used to determine a consistent estimator of the vector

$$
T(x)=\left\{T_{1}(x), T_{2}(x)\right\}
$$


becomes of the following form:

$$
z^{T} \cdot \Delta \cdot C \cdot z=\hat{T}_{n}^{(1)}(x), \quad z^{T} \cdot \Delta \cdot C^{2} \cdot z=\hat{T}_{n}^{(2)}(x) .
$$

Applying the results of [10] we prove that, for an arbitrary real number $x$ and for any $i=1,2$, the estimator $\hat{T}_{n}^{(i)}$ converges in probability,

$$
\hat{T}_{n}^{(i)}(x) \rightarrow T^{(i)}(x)
$$

Next we study the solutions of the following system of equations:

$$
\left\{\begin{array}{l}
z^{T} \cdot \Delta \cdot C \cdot z=T^{(1)}(x), \\
z^{T} \cdot \Delta \cdot C^{2} \cdot z=T^{(2)}(x) .
\end{array}\right.
$$

The system of equations (5) is solvable, since

$$
\left\{z_{1}, z_{2}\right\}=\left\{T_{1}(x), T_{2}(x)\right\}
$$

is one of its solutions.

Let

$$
\hat{\Delta}=\operatorname{diag}\left\{\sqrt{c_{1}}, \sqrt{c_{2}}\right\},
$$

and let the entries of the orthogonal matrix $U=\left\|u_{i j}\right\|, i \in\{1,2\}, j \in\{1,2\}$, be defined as follows:

$$
\begin{aligned}
u_{11} & =\frac{\sqrt{\beta}}{\sqrt{\alpha+\beta}}, & u_{12} & =\frac{\sqrt{\alpha}}{\sqrt{\alpha+\beta}}, \\
u_{21} & =-\frac{\sqrt{\alpha}}{\sqrt{\alpha+\beta}}, & u_{22} & =\frac{\sqrt{\beta}}{\sqrt{\alpha+\beta}} .
\end{aligned}
$$

Now we change twice the variables in the system of equations (5): first

$$
\hat{z}=\hat{\Delta} \cdot z
$$

and then

$$
y=U^{-1} \cdot \hat{z} .
$$

As a result we obtain the following system of equations:

$$
y_{1}^{2}+b \cdot y_{2}^{2}=T^{(1)}(x), \quad y_{1}^{2}+b^{2} \cdot y_{2}^{2}=T^{(2)}(x) .
$$

Then we represent the matrices $C$ and $C^{(2)}$ as

$$
C=G_{1}+b \cdot G_{2}, \quad C^{(2)}=G_{1}+b^{2} \cdot G_{2} .
$$

Note that $G_{1}=\left\|g_{i j}^{(1)}\right\|, i \in\{1,2\}, j \in\{1,2\}$, where

$$
\begin{array}{ll}
g_{11}^{(1)}=c_{1}, & g_{12}^{(1)}=c_{2}, \\
g_{21}^{(1)}=c_{1}, & g_{22}^{(1)}=c_{2},
\end{array}
$$

and $G_{2}=\left\|g_{i j}^{(2)}\right\|, i \in\{1,2\}, j \in\{1,2\}$, and where

$$
\begin{aligned}
& g_{11}^{(2)}=c_{1}, \quad g_{12}^{(2)}=-c_{2}, \\
& g_{21}^{(2)}=-c_{1}, \quad g_{22}^{(2)}=c_{2} .
\end{aligned}
$$

Hence

$$
A=\hat{\Delta} \cdot C \cdot \hat{\Delta}^{-1}=\hat{G}_{1}+b \cdot \hat{G}_{2}
$$

and the entries of the matrix $\hat{G}_{1}=\left\|\hat{g}_{i j}^{(1)}\right\|, i \in\{1,2\}, j \in\{1,2\}$, are obtained from the equalities

$$
\begin{aligned}
& \hat{g}_{11}^{(1)}=c_{1}, \quad \hat{g}_{12}^{(1)}=\sqrt{c_{1} \cdot c_{2}}, \\
& \hat{g}_{21}^{(1)}=\sqrt{c_{1} \cdot c_{2}}, \quad \hat{g}_{22}^{(1)}=c_{2} .
\end{aligned}
$$


Similarly, the entries of the matrix $\hat{G}_{2}=\left\|\hat{g}_{i j}^{(2)}\right\|, i \in\{1,2\}, j \in\{1,2\}$, are obtained from the equalities

$$
\begin{aligned}
& \hat{g}_{11}^{(2)}=c_{1}, \quad \hat{g}_{12}^{(2)}=-\sqrt{c_{1} \cdot c_{2}}, \\
& \hat{g}_{21}^{(2)}=-\sqrt{c_{1} \cdot c_{2}}, \quad \hat{g}_{22}^{(2)}=c_{2} .
\end{aligned}
$$

Consider the vector-column $T(x)$ with the coordinates $T_{1}(x)$ and $T_{2}(x)$. Since

$$
z_{1}=T_{1}(x), \quad z_{2}=T_{2}(x)
$$

are solutions of the system of equations (5), we have

$$
T(x)^{T} \cdot A \cdot T(x)=T^{(1)}(x), \quad T(x)^{T} \cdot A^{2} \cdot T(x)=T^{(2)}(x) .
$$

Put

$$
v_{1}(x)=T(x)^{T} \cdot \hat{G}_{1} \cdot T(x), \quad v_{2}(x)=T(x)^{T} \cdot \hat{G}_{2} \cdot T(x) .
$$

Since the coordinates of the vector $T(x)$ are nonnegative, we easily get the inequality

$$
v_{1}(x)>v_{2}(x) .
$$

On the other hand,

$$
v_{1}(x)+b \cdot v_{2}(x)=T^{(1)}(x), \quad v_{1}(x)+b^{2} \cdot v_{2}(x)=T^{(2)}(x),
$$

that is

$$
\frac{T^{(2)}(x)-b \cdot T^{(1)}(x)}{1-b}=g_{1}(x)>g_{2}(x)=\frac{T^{(1)}(x)-T^{(2)}(x)}{b \cdot(1-b)} .
$$

Solving the system of equations (5) and applying inequalities (6) to its positive solutions, we conclude that:

(i) if

$$
\beta<B_{1}=\frac{T^{(1)}(x)-T^{(2)}(x)}{T^{(1)}(x) \cdot(1+b)-T^{(2)}(x)},
$$

then the system of equations (5) has a unique positive solution $z^{(1)}=\left\{z_{1}^{(1)}, z_{2}^{(1)}\right\}$, where

$$
\begin{aligned}
& z_{1}^{(1)}=\sqrt{\frac{T^{(2)}(x)-b \cdot T^{(1)}(x)}{\alpha+\beta}}+\sqrt{\frac{c_{2}}{c_{1}}} \cdot \sqrt{\frac{T^{(1)}(x)-T^{(2)}(x)}{b \cdot(\alpha+\beta)}}, \\
& z_{2}^{(1)}=\sqrt{\frac{T^{(2)}(x)-b \cdot T^{(1)}(x)}{\alpha+\beta}}-\sqrt{\frac{c_{1}}{c_{2}}} \cdot \sqrt{\frac{T^{(1)}(x)-T^{(2)}(x)}{b \cdot(\alpha+\beta)}} ;
\end{aligned}
$$

(ii) if

$$
\beta>B_{2}=\frac{b \cdot\left(T^{(2)}(x)-b \cdot T^{(1)}(x)\right)}{T^{(1)}(x) \cdot(1+b)-T^{(2)}(x)},
$$

then there exists only one positive solution $z^{(2)}=\left\{z_{1}^{(2)}, z_{2}^{(2)}\right\}$ among the solutions of the system of equation (5), where

$$
\begin{aligned}
& z_{1}^{(2)}=\sqrt{\frac{T^{(2)}(x)-b \cdot T^{(1)}(x)}{\alpha+\beta}}-\sqrt{\frac{c_{2}}{c_{1}}} \cdot \sqrt{\frac{T^{(1)}(x)-T^{(2)}(x)}{b \cdot(\alpha+\beta)}}, \\
& z_{2}^{(2)}=\sqrt{\frac{T^{(2)}(x)-b \cdot T^{(1)}(x)}{\alpha+\beta}}+\sqrt{\frac{c_{1}}{c_{2}}} \cdot \sqrt{\frac{T^{(1)}(x)-T^{(2)}(x)}{b \cdot(\alpha+\beta)}} ;
\end{aligned}
$$


(iii) if

$$
B_{1}<\beta<B_{2},
$$

then both solutions are positive.

Consider the following random vector $y_{n}(x)=\left\{y_{n 1}(x), y_{n 2}(x)\right\}$ whose coordinates are determined from equalities

$$
\begin{gathered}
y_{n 1}(x)=\sqrt{\frac{\hat{T}_{n}^{(2)}(x)-b \cdot \hat{T}_{n}^{(1)}(x)}{\alpha+\beta}}, \\
y_{n 2}(x)=\sqrt{\frac{\hat{T}_{n}^{(1)}(x)-\hat{T}_{n}^{(2)}(x)}{b \cdot(\alpha+\beta)}} .
\end{gathered}
$$

Taking into account relations (7), (8), and (9) together with the convergence (4) of the estimators

we prove the following assertion.

$$
\left\{\hat{T}_{n}^{(1)}(x), \hat{T}_{n}^{(2)}(x)\right\}
$$

Theorem 2.2. Assume that

1) $\alpha+\beta \neq 1$,

2) $\alpha \neq \beta$.

Then, for all $x \in(-\infty, \infty)$, such that

$$
T_{1}(x)>0 \text { and } T_{2}(x)>0,
$$

the coordinates of the random vector $y_{n}(x)=\left\{y_{n 1}(x), y_{n 2}(x)\right\}$ are real numbers with the probability that approaches one as $n \rightarrow \infty$. Moreover,

(i) if $\beta<B_{1}$, then the vector

$$
\hat{y}_{n}^{(1)}=\left\{y_{n 1}(x)+\sqrt{\frac{\alpha}{\beta}} \cdot y_{n 2}(x) ; y_{n 1}(x)-\sqrt{\frac{\beta}{\alpha}} \cdot y_{n 2}(x)\right\}
$$

is a consistent estimator of the vector $T(x)=\left\{T_{1}(x), T_{2}(x)\right\}$;

(ii) if $\beta>B_{2}$, then the vector

$$
\hat{y}_{n}^{(2)}=\left\{y_{n 1}(x)-\sqrt{\frac{\alpha}{\beta}} \cdot y_{n 2}(x) ; y_{n 1}(x)+\sqrt{\frac{\beta}{\alpha}} \cdot y_{n 2}(x)\right\}
$$

is a consistent estimator of the vector $T(x)=\left\{T_{1}(x), T_{2}(x)\right\}$;

(iii) if $B_{1}<\beta<B_{2}$, then one of the vectors, either $\hat{y}_{n}^{(1)}$ or $\hat{y}_{n}^{(2)}$, is a consistent estimator of the vector

$$
T(x)=\left\{T_{1}(x), T_{2}(x)\right\} .
$$

Remark 2.1. According to Theorem 2.2, the consistent estimator of the vector $T(x)$ is not necessarily unique, that is several solutions of the system of equations (3) can be consistent estimators. It is shown in the paper [10] that, in the case of a general "hidden Markov model"

$$
(\zeta(k), \eta(k)), \quad k=0,1,2, \ldots,
$$

with the set of states $E=\{1,2, \ldots, m\}$, a consistent estimator of the vector $T(x)$ can be distinguished in the set of solutions of the system of equations (3) with the help of the statistics $\hat{T}_{n}^{(0)}(x)$ if

$$
p_{i_{1}} \cdot T_{i_{1}}^{(0)}+\cdots+p_{i_{r}} \cdot T_{i_{r}}^{(0)} \neq 0
$$


for all $1 \leq r \leq m, i_{1}, i_{2}, \ldots, i_{r} \in E=\{1,2, \ldots, m\}$, where

$$
\begin{gathered}
p=\left\{p_{1}, p_{2}, \ldots, p_{m}\right\}=U^{T} \cdot \hat{\Delta} \cdot c, \\
T^{(0)}(x)=\left\{T_{1}^{(0)}(x), T_{2}^{(0)}(x), \ldots T_{2}^{(0)}(x)\right\}=U^{T} \cdot \hat{\Delta} \cdot T(x) .
\end{gathered}
$$

Condition (10) does not hold in case considered in Theorem 2.2. Indeed,

$$
\begin{gathered}
p=\left\{p_{1}, p_{2}\right\}=U^{T} \cdot \hat{\Delta} \cdot c=\{1,0\}, \\
T^{(0)}(x)=\left\{T_{1}^{(0)}(x), T_{2}^{(0)}(x)\right\}=U^{T} \cdot \hat{\Delta} \cdot T(x) \\
=\left\{c_{1} \cdot T_{1}(x)+c_{2} \cdot T_{2}(x), \sqrt{c_{1} \cdot c_{2}} \cdot\left(T_{2}(x)-T_{1}(x)\right)\right\},
\end{gathered}
$$

whence

$$
p_{2} \cdot T_{2}^{(0)}(x)=0
$$

This means that the statistics $\hat{T}_{n}^{(0)}(x)$ is useless when distinguishing a consistent estimator. On the other hand, a consistent estimator of the vector $T(x)$ can be chosen between two candidates proposed by Theorem 2.2 if one uses one of the following two statistics:

$$
\begin{gathered}
\hat{T}_{n}^{(s, r)}(x)=\frac{1}{n} \sum_{t=1}^{n} \chi[\tau(t)<x, \tau(t+s)<x, \tau(t+s+r)<x] ; \\
1 \leq s \leq 2, \quad 1 \leq r \leq 2 .
\end{gathered}
$$

This result follows from Lemma 4 of the paper [10].

Remark 2.2. If assumption 1 of Theorem 2.2 does not hold and thus $\alpha+\beta=1$, then the stochastic process $\tau(t), t=1,2, \ldots$, is a sequence of independent random variables and hence one can construct an estimator for the mixture

$$
c_{1} \cdot T_{1}(x)+c_{2} \cdot T_{2}(x)
$$

of the distribution functions $T_{1}(x)$ and $T_{2}(x)$ from the observations $\{\tau(1), \ldots, \tau(n+2)\}$ after the second component of the two dimensional process $(c(t), \tau(t)), t=1,2, \ldots$.

Remark 2.3. If assumption 2 of Theorem 2.2 does not hold, that is if $\alpha=\beta$, then one nevertheless can construct the estimators of unknown distribution functions $T_{1}(x)$ and $T_{2}(x)$ by using the vector of observations $\{\tau(1), \ldots, \tau(n+2)\}$ after the second component of the two dimensional stochastic process $(c(t), \tau(t)), t=1,2, \ldots$. On the other hand, one cannot associate these estimators with the states of the Markov chain $c(t)$ anymore.

We use Theorem 2.2 for a compound Poisson process governed by a simple Markov regenerative process. More precisely, assume that two families of random variables

$$
\Xi=\left\{\xi_{k}, k=1,2, \ldots\right\}, \quad Z=\left\{\zeta_{k}, k=1,2, \ldots\right\}
$$

are defined on the same probability space $(\Omega, \Im, P)$ together with the following two stochastic processes:

(1) a simple Markov regenerative process $x(t), t \geq 0$, with parameters $(\mu, \nu)$; the process $x(t)$ is assumed to be independent of the families of random variables $\Xi$ and $Z$; its set of states is $E=\{1,2\}$; moreover, both sojourn times are assumed to be exponential with parameter $\mu$ for the state $\{1\}$, and with parameter $\nu$ for the state $\{2\}$;

(2) a Poisson process $\Pi(t), t \geq 0$, with parameter $\lambda$; the process is assumed to be independent of the families of random variables $\Xi$ and $Z$, and to be independent of the stochastic process $x(t), t \geq 0$.

Further, let $S(0)=0$, and let $S(k), k=1,2, \ldots$, denote a random variable with the Erlang distribution with parameters $(k, \lambda)$. 
Definition 2.2. A two dimensional stochastic process $(\xi(t), \zeta(t)), t \geq 0$,

$$
\xi(t)=\sum_{k=1}^{\Pi(t)} \xi_{k} \cdot \chi[x(S(k))=1], \quad \zeta(t)=\sum_{k=1}^{\Pi(t)} \zeta_{k} \cdot \chi[x(S(k))=2]
$$

is called a compound Poisson process governed by a simple Markov regenerative process.

Consider the following stochastic process

$$
\theta(k)=\xi_{k} \cdot \chi[x(S(k))=1]+\zeta_{k} \cdot \chi[x(S(k))=2], \quad k=1,2, \ldots .
$$

Lemma 2.1. The stochastic process with discrete time $\theta(k), k=1,2, \ldots$, given by (11) corresponds to the following scheme of independent random variables defined on the Markov chain:

$$
\theta(k)=\theta_{\eta(k)}^{(k)}, \quad k=1,2, \ldots,
$$

where $\eta(k), k=1,2, \ldots$, is a homogeneous Markov chain with the set of states

$$
E=\{1,2\} \text {. }
$$

The matrix of one step transition probabilities $P=\left\|p_{i j}\right\|, i, j=1,2$, of the chain $\theta_{k}$ is defined as

$$
\begin{array}{ll}
p_{11}=\frac{\lambda+\nu}{\lambda+\mu+\nu}, & p_{12}=\frac{\mu}{\lambda+\mu+\nu}, \\
p_{21}=\frac{\nu}{\lambda+\mu+\nu}, & p_{22}=\frac{\lambda+\mu}{\lambda+\mu+\nu} .
\end{array}
$$

Here $\left[\left\{\theta_{i}^{(k)}, k=0,1,2, \ldots\right\}, i=1,2\right]$ is a family of jointly independent random variables that are independent of the Markov chain $\eta(k)$ and such that the distribution of $\theta_{1}^{(k)}$ does not depend on the index $k$ and coincides with the distribution of $\xi_{k}$, while the distribution of the random variable $\theta_{2}^{(k)}$ does not depend on the index $k$ and coincides with the distribution of $\zeta_{k}$.

Proof. Comparing equalities (11) and (12), we deduce that

$$
\theta(k)=x(S(k)), \quad k=1,2, \ldots .
$$

Using the properties of the Poisson process $\Pi(t)$ and the stochastic structure of the regenerative process $x(t)$, we obtain

$$
\begin{aligned}
p_{i j} & =\mathrm{P}\{\eta(k+1)=j / \eta(k)=i\}=\mathrm{P}\{x(S(1))=j / \eta(0)=i\} \\
& =\lambda \int_{0}^{\infty} e^{-\lambda \cdot t} \mathrm{P}\{x(t)=j / x(0)=i\} d t=\lambda \tilde{p}_{i j}(\lambda), \quad i, j=1,2 .
\end{aligned}
$$

Here $\tilde{p}_{i j}(s)$ denotes the Laplace transform of the transition function of the Markov process $x(t)$, that is

where

$$
\tilde{p}_{i j}(s)=\int_{0}^{\infty} e^{-\lambda \cdot t} p_{i j}(t) d t
$$

$$
p_{i j}(t)=\mathrm{P}\{x(t)=j / x(0)=i\} .
$$

The transition functions $p_{i j}(t), i, j=1,2$, of the Markov process $x(t)$ satisfy the following Kolmogorov system of the differential equations

$$
\begin{aligned}
& \frac{d p_{11}(t)}{d t}=-\mu \cdot p_{11}(t)+\nu \cdot p_{12}(t), \\
& \frac{d p_{22}(t)}{d t}=-\mu \cdot p_{22}(t)+\nu \cdot p_{21}(t) .
\end{aligned}
$$


Evaluating the Laplace transform for each of these equations and using equalities (12) we get

$$
\begin{aligned}
& p_{11}-1=-\frac{\mu}{\lambda} \cdot p_{11}+\frac{\nu}{\lambda} \cdot p_{12}, \\
& p_{22}-1=-\frac{\mu}{\lambda} \cdot p_{22}+\frac{\nu}{\lambda} \cdot p_{21},
\end{aligned}
$$

respectively. Since $P=\left\|p_{i j}\right\|, i, j=1,2$, is a stochastic matrix, we complete the proof of Lemma 2.1.

\section{Concluding Remarks}

Let

$$
\{\theta(1), \theta(2), \ldots, \theta(n+2)\}
$$

be the vector of observations after the stochastic process $\theta(k), k=1,2, \ldots$. Assume that the distribution functions

$$
K(x)=\mathrm{P}\left\{\xi_{k}<x\right\} \quad \text { and } \quad L(x)=\mathrm{P}\left\{\zeta_{i}<x\right\}
$$

of the families $\Xi$ and $Z$ are unknown. The functions $K(x)$ and $L(x)$ can be estimated explicitly by Lemma 2.1 and Theorem 2.2 by using the statistics

$$
\hat{q}_{n}^{(i)}(x)=\frac{1}{n} \sum_{t=1}^{n} \chi[\theta(t)<x, \theta(t+i)<x], \quad i=1,2,
$$

instead of statistics $\hat{T}_{n}^{(i)}(x), i=1,2$, and by putting

$$
\alpha=\frac{\mu}{\lambda+\mu+\nu}, \quad \beta=\frac{\nu}{\lambda+\mu+\nu} .
$$

Note that the assumptions of Theorem 2.2 hold in view of the definition of the Markov regenerative process $x(t)$. First, the equality $\alpha=\beta$ is not possible, it means that $\mu=\nu$, that is $x(t), t \geq 0$, is not a regenerative process but is a simple Poisson process with parameter $\mu$. Since

condition $\alpha+\beta \neq 1$ also holds.

$$
0<\frac{\mu+\nu}{\lambda+\mu+\nu}<1
$$

\section{BIBLIOGRAPHY}

1. V. V. Anisimov, Random Processes with a Discrete Component. Limit Theorems, "Vyshcha Shkola", Kiev, 1988. (Russian) MR955492 (89m:60003)

2. A. A. Vol̆na, Asymptotic optimization for stochastic models based on a compound Poisson process, Kibernetika i Sistemnyi Analiz (2011), no. 4, 11-23; English transl. in Cybernetics and System Analysis (2011), no. 4, 649-658.

3. I. I. Gikhman and A. V. Skorokhod, The Theory of Stochastic Processes, vol. 1, "Nauka", Moscow, 1971; vol. 2, "Nauka", Moscow, 1973; English transl., vol. 1, Springer-Verlag, New York-Heidelberg, 1974; vol. 2, Springer-Verlag, New York-Heidelberg, 1975.

4. I. N. Kovalenko, Analysis of Rare Events for Estimating the Efficiency and Reliability of Systems, "Sovetskoe Radio", Moscow, 1980. (Russian)

5. D. R. Cox and P. A. W. Lewis, The Statistical Analysis of Series of Events, Methuen \& Co., Ltd. and John Wiley \& Sons, Inc., London and New York, 1966. MR0199942 (33:8082)

6. V. S. Korolyuk and A. F. Turbin, Mathematical Foundations of the State Lumping of Large Systems, "Naukova Dumka", Kiev, 1978; English transl., Kluwer Academic Publishers Group, Dordrecht, 1993. MR1281385 (95e:60071)

7. D. S. Sil'vestrov, Semi-Markov Processes with a Discrete State Space, "Sovetskoe Radio", Moscow, 1980. (Russian) MR612026 (82k:60181)

8. M. Tim Jones, Al Application Programming, Charles River Media, Inc, Hingham, Massachusetts, 2003.

9. L. R. Rabiner and B. H. Juang, An introduction to hidden Markov models, IEEE ASSP Mag. 3 (1986), no. 1, 4-16. 
10. A. A. Voinna, Statistical estimation in a scheme of random variables on a Markov chain in the presence of incomplete observations, Teor. Veroyatnost. i Mat. Statist. 37 (1987), 16-26, 134; English transl. in Theor. Prob. Math. Statist. 37 (1988), 19-28. MR913905 (88k:62164)

11. A. A. Voĭna and E. Czapla, An application of the correlation structure of a Markov chain for the estimation of shift parameters in queuing systems, Teor. Imovir. Mat. Stat. v71 (2004), 49-56; English transl. in Theor. Prob. Math. Statist. 71 (2005), 53-61.

12. A. Wojna, Ryzyko w Procesach Finansowych Oraz Metody Badania Koniunktury, PK, Koszalin, 2009.

Department of Economics and Management, Koszalin University of Technology, E. KwiatKowskiego Street, 6E, Koszalin, 75-343, Poland

E-mail address: avoina@hotmail.com

Received 09/SEP/2010

Translated by S. KVASKO 